\title{
LINEAR FUNCTIONALS ON ORLICZ SEQUENCE SPACES WITHOUT LOCAL CONVEXITY
}

\author{
MARIAN NOWAK \\ Institute of Mathematics \\ A. Mickiewicz University \\ Matejki 48/49, 60-769 Poznan \\ Poland \\ (Received March 8, 1990)
}

\begin{abstract}
The general form of continuous linear functionals on an Orlicz sequence space $1^{\phi}$ (nonseparable and non-locally convex in general) is obtained. It is proved that the space $h^{\dagger}$ is an $M$-ideal in $1^{\dagger}$.
\end{abstract}

KEY WORDS AND PHRASES. Orlicz sequence spaces, Köthe dual, Riesz spaces, Mackey topologies, modular spaces, and $M$-ideals.

1991 AMS SUBJECT CLASSIFICATION CODE. Primary 46 E 30.

INTRODUCTION. The general form of continuous linear functionals on an Orlicz space $L \downarrow$, defined by a convex Orlicz function $\phi$ has been found by Ando [2] (for $\phi$ being an $N$-function and for a finite measure space) and by Rao [21], Fernandez [7] (for $\phi$ being a Young function and for a general measure space).

In this paper we describe the dual space $\left(1^{\dagger}\right)^{*}$ of an Orlicz sequence space $1^{\phi}$ defined by an arbitrary Orlicz function $\phi$ (not necessarily convex) such that $\phi(u) / u \rightarrow \infty$ as $u \rightarrow \infty$. For this purpose we shall first use the description of the Mackey topology $\tau_{\phi}$ of $1^{\dagger}$, obtained by Kalton [8], when $\phi$ satisfies the $\Delta_{2}$-condition at 0 , and by Drewnowski and Nawrocki [5], in general. The Mackey topology $\tau_{\phi}$ is normable and we consider two natural norms on $1^{\phi}$ which generate $\tau_{\phi}$. Thus we can define two corresponding norms in $\left(1^{\phi}\right)^{*}$. Moreover, we consider $1^{\text {t }}$ from the point of view of the theory of modular spaces (see [15], [16], [17]). We investigate the conjugate modular (in the sense of Nakano [17]) on $\left(1^{\circ}\right)^{\circ}$ and consider two other norms on $\left(1^{\dagger}\right)^{*}$ defined in a natural way by the conjugate modular. It is well-known that $\left(1^{\dagger}\right)^{*}=\left(1^{\dagger}\right)_{n}^{-}+\left(1^{\dagger}\right)_{s}^{-}$, where $\left(1^{\phi}\right)_{n}^{-}$and $\left(1^{\dagger}\right)_{s}^{-}$denote the sets of all order continuous and singular linear functionals on $1^{\phi}$ respectively. We first show that the Köthe dual $\left(1^{\dagger}\right)^{x}$ of $1^{\phi}$ coincides with the Orlicz sequence space $1^{\phi^{*}}$, where $\phi^{*}$ denotes the complementary function of $\varphi$ in the sense of Young. Thus we obtain the corresponding characterization of $\left(1^{\phi}\right)_{n}^{-}$. Next, we prove that the conjugate modular and all four norms defined on $\left(1^{\dagger}\right)^{*}$ coincide on $\left(1^{\phi}\right)_{s}^{-}$. Following the idea of [2] we construct a Riesz isometric isomorphism of $\left(1^{\star}\right)_{s}^{-}$ onto some Riesz subspace $B_{\phi}(\mathrm{N})$ (dependent on $\phi$ ) of the Banach lattice $b \mathrm{a}(\mathrm{N})$ of all real-valued bounded finitely additive set functions on $N$. We prove that there exists an isometric isomorphism of the Banach space $\left(\left(1^{\dagger}\right)^{*},\|\cdot\|_{\phi}^{*}\right)$ (for the definition of the norm $\|\cdot\|_{\phi}^{*}$ see section 2) onto the Banach space $1^{* *} \times B_{\phi}(N)$ given by the mapping $f \rightarrow(y, v)$ such that $f(x)=\sum_{i=1}^{\infty} x(i) y(i)+\int x d v$ for all $x \in 1^{\star}$ and $\|f\|_{\phi}^{*}=\|y\|_{\phi^{*}}+|v|(N)$. From this it follows that $h^{\phi}$ (the ideal of elements of absolutely continuous $F$-norm on $1^{\dagger}$ ) is an $M$-ideal of $1^{\dagger}$ (see [3, definition 2.1]). As an application, we obtain that every continuous linear function on $h^{\phi}$ has the unique norm preserving extension to $1^{\phi}$.

1. Preliminaries. For terminology concerning locally solid Riesz spaces we refer to [1] and [14]. For a Riesz space $(E, z)$ let $E^{+}=\{u \in E: u \geq 0\}$ (the positive cone of $E$ ). By $\mathbf{N}$ we will denote the set of all natural numbers. Denote by $\omega$ the space of all real-valued sequences. For the sequence $x, x(i)$ means the 
$i$-th coordinate of $x$, and we shall denote by $x^{(n)}$ the $n$-th section of $x$ (that is $x^{(n)}(i)=x(i)$ for $i \leq n, x^{(n)}(i)=0$ for $i>n)$. For a subset $A$ of $N$ we will denote by $x_{A}$ the sequence such that $x_{A}(i)=x(i)$ for $i \in A$ and $x_{A}(i)=0$ for $i \notin A$. If $f$ is a linear functional on a subspace $X$ of $\omega$, we will denote by $f_{A}$ the functional defined as: $f_{A}(x)=f\left(x_{A}\right)$ for $x \in X$. It is known that $\omega$ is a super Dedekind complete Riesz space under the ordering $x \leq y$ whenever $x(i) \leq y(i)$ for $i \in \mathrm{N}$.

Now we recall some terminology concerning Orlicz sequence spaces (see [11], [12], [22], and [25]).

By an Orlicz function $\phi$ we mean a function $\phi:[0, \infty) \rightarrow[0, \infty)$ which is non-decreasing, continuous for $u \geq 0$ and $\phi(u)=0$ iff $u=0$. Throughout this paper we shall assume that $\varphi$ satisfies the following condition: $\phi(u) / u \rightarrow \infty$ as $u \rightarrow \infty$. Every Orlicz function $\phi$ determines the functional $\rho_{\phi}: \omega \rightarrow[0, \infty]$ defined by the formula:

$$
\rho_{\phi}(x)=\sum_{i=1}^{\infty} \phi(|x(i)|) .
$$

Then $1^{\dagger}=\left\{x \in \omega: \rho_{\phi}(\lambda x)<\infty\right.$ for some $\left.\lambda>0\right\}$ is called an Orlicz sequence space defined by $\phi$. The space $1^{\star}$ is an ideal of $\omega$ and the functional $\rho_{\phi}$ restricted to $1^{\phi}$ is an orthogonal additive modular, i.e., $\rho_{\phi}$ satisfies the following conditions:

(1) $\rho_{\phi}(x)=0$ iff $x=0$.

(2) $\quad \rho_{\phi}\left(x_{1}\right) \leq \rho_{\phi}\left(x_{2}\right)$ if $\left|x_{1}\right| \leq\left|x_{2}\right|$.

(3) $\rho_{\phi}(\lambda x) \rightarrow 0$ if $\lambda \rightarrow 0$.

(4) $\quad \rho_{\phi}\left(x_{1}+x_{2}\right)=\rho_{\phi}\left(x_{1}\right)+\rho_{\phi}\left(x_{2}\right)$ if $\left|x_{1}\right| \wedge\left|x_{2}\right|=0$.

These conditions imply that $\rho_{\phi}\left(x_{1} \vee x_{2}\right) \leq \rho_{\phi}\left(x_{1}\right)+\rho_{\phi}\left(x_{2}\right)$ for $x_{1}, x_{2} \geq 0$. Moreover, $\rho_{\phi}$ satisfies the following axiom of completeness (see [15]):

(C) If $x_{n} \geq 0$ for $n=1,2, \ldots$ and $\sum_{n=1}^{\infty} \rho_{\phi}\left(x_{n}\right)<\infty$, then there exists $y \in 1^{\dagger}$ such that $y=\sup x_{n}$ and $\rho_{\phi}(y) \leq \sum_{n=1}^{\infty} \rho_{\phi}\left(x_{n}\right)$.

If $\phi$ is a convex Orlicz function, then the modular $\rho_{\phi}$ is convex, i.e.,

$$
\rho_{\phi}\left(a x_{1}+b x_{2}\right) \leq a \rho_{\phi}\left(x_{1}\right)+b \rho_{\phi}\left(x_{2}\right) \text { for } a, b \geq 0 \text { with } a+b=1 \text {. }
$$

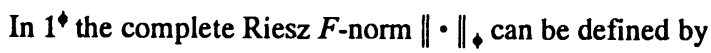

$$
|x|_{\downarrow}=\inf \left\{\lambda>0: \rho_{\phi}(x / \lambda) \leq \lambda\right\} \text {. }
$$

We shall denote by $\tau_{\phi}$ the topology of the $F$-norm $|\cdot|_{\phi}$. Let $h^{\phi}=\left\{x \in 1^{\phi}: \rho_{\phi}(\lambda x)<\infty\right.$ for all $\left.\lambda>0\right\}$. Then $h^{\dagger}$ is the ideal of elements of absolutely continuous $F$-norm $|\cdot|_{\text {, on }} 1^{\phi}$.

We say that $\phi$ satisfies the $\Delta_{2}$-condition at 0 , whenever $\limsup _{\mu \rightarrow 0} \phi(2 u) / \phi(u)<\infty$. It is known that $1^{\phi}=h^{\phi}$ (i.e. $1^{\dagger}$ is separable) iff $\phi$ satisfies the $\Delta_{2}$-condition at 0 .

We say that two Orlicz functions $\phi$ and $\psi$ are equivalent at 0 , in symbols $\phi \sim \psi$, if there exist positive numbers a,b,c,d and $u_{0}>0$ such that a $\phi(b u) \leq \psi(u) \leq c \phi(d u)$ for $0 \leq u \leq u_{0}$. It is well-known that if $\phi \sim \psi$ then $1^{\phi}=1^{\psi}$ and $\tau_{\phi}=\tau_{\psi}$. Moreover, the space $\left(1^{\phi}, \tau_{\phi}\right)$ is locally convex iff there exists a convex Orlicz function $\psi$ such that $\phi \sim \psi$ (see [25], Theorem 3.1.5]. Separable Orlicz sequence spaces without local convexity have been investigated in detail by Kalton [8]. For examples of non-separable and non-locally convex Orlicz sequence spaces see [5].

We denote by $p_{\phi}$ the Minkowski functional of the absolutely convex absorbing subset $k^{\natural}=\left\{x \in \omega: \rho_{\phi}(x)<\infty\right\}$ of $1^{\downarrow}$. Thus

$$
p_{\phi}(x)=\inf \left\{\lambda>0: \rho_{\phi}(x / \lambda)<\infty\right\}
$$

for all $x \in 1^{\star}, p_{\phi}(x) \leq|x|$, for $x \in 1^{\phi}$, and $h^{\dagger}=\operatorname{ker} p_{\phi}$. 
2. Norms on the dual space $\left(1^{\dagger}\right)^{*}$ of $1^{\downarrow}$. In this section we define in two different ways some natural norms on $\left(1^{\dagger}\right)^{*}$. For this purpose we shall first use the description of the Mackey topology of $\left(1^{\dagger}, \tau_{\phi}\right)$ given in [5], and next, we apply the Nakano's theory of conjugate modulars [17].

Let us put

$$
\dot{\phi}^{*}(v)=\sup \{u v-\phi(u): u \geq 0\} \text { for } v \geq 0 .
$$

Then $\phi^{\bullet}$ will be called the function complementary to $\phi$ in the sense of Young. It is seen that $\phi^{\bullet}$ is a convex function, taking only finite values, and $\dot{\phi}^{*}(0)=0$. This means that $\dot{\phi}^{*}$ is a Young function (see [12], [13], [26]). The additional properties of $\phi^{*}$ are included in the following

LEMMA2.1. (a) If $\liminf _{u \rightarrow 0} \phi(u) / u=0$, then $\phi^{*}$ vanishes only at 0 and $\lim _{v \rightarrow 0} \phi^{*}(v) / v=0, \lim _{v \rightarrow \infty} \phi^{*}(v) / v=\infty$ (i.e. $\phi^{*}$ is an $N$-function in the sense of [11]).

(b) If $\liminf _{u \rightarrow 0} \phi(u) / u>0$, then $\phi^{*}$ vanishes near zero and $\lim _{v \rightarrow \infty} \phi^{*}(v) / v=\infty$ (i.e. $1^{+}=1^{\infty}$ ).

PROOF. (a) We can easily verify that $\phi^{\circ}(v)>0$ for $v>0$. In the same way as in $[4, \S 2]$ we can show that $\lim _{v \rightarrow 0} \phi^{*}(v) / v=0$ and $\lim _{v \rightarrow \infty} \phi^{*}(v) / v=\infty$.

(b) We shall show that there exists $v_{0}>0$ such that $\phi^{\circ}(v)=0$ for $0 \leq v \leq v_{0}$, and $\phi^{\circ}(v)>0$ for $v>v_{0}$. indeed, since $\liminf _{u \rightarrow 0} \phi(u) / u>0$ there exist numbers $v^{\prime}>0$ and $u^{\prime}>0$ such that $u v^{\prime} \leq \phi(u)$ for $0 \leq u \leq u^{\prime}$, and since $\lim _{u \rightarrow \infty} \phi(u) / u=\infty$ (by our assumption) there exists a number $u^{\prime \prime}>0$ with $u^{\prime \prime}>u^{\prime}$ such that $u \leq \phi(u)$ for $u \geq u^{\prime \prime}$. Taking $\mathrm{v}^{\prime \prime}>0$ such that $1 / \mathrm{v}^{\prime \prime}=\sup \left\{u / \phi(u): u^{\prime} \leq u \leq u^{\prime \prime}\right\}$, we have $u \mathrm{v}^{\prime \prime} \leq \phi(u)$ for $u^{\prime} \leq u \leq u^{\prime \prime}$. Then for $\mathrm{v}_{1}=\min \left(1, \mathrm{v}^{\prime}, \mathrm{v}^{\prime \prime}\right)$ we get $u \mathrm{v}_{1} \leq u \mathrm{v}^{\prime} \leq \phi(u)$ for $u \geq u^{\prime \prime}, u v_{1} \leq u \mathrm{v}^{\prime \prime} \leq \phi(u)$ for $u^{\prime} \leq u \leq u^{\prime \prime}$, and $u v_{1} \leq u \leq \phi(u)$ for $u \geq u^{\prime \prime}$. Hence $u v_{1}-\phi(u) \leq 0$ for $u \geq 0$, so that $\phi^{\prime \prime}\left(v_{1}\right)=0$. On the other hand, there exists a number $v_{2}>0$ such that $\phi^{*}\left(v_{2}\right)>0$. Since $\phi^{*}$ is convex, there exists a number $v_{0}>0$ such that $\phi^{*}(v)=0$ for $0 \leq v \leq v_{0}$, and $\phi^{*}(v)>0$ for $v>v_{0}$. Moreover, as in $[4, \S 2]$ we can show that $\lim _{v \rightarrow \infty} \phi^{*}(v) / v=\infty$.

For an Orlicz function $\phi$ we shall denote by $\hat{\phi}$ the convex minorant of $\phi$ in a neighborhood of 0 , i.e., $\hat{\phi}$ is the largest Orlicz function such that $\hat{\phi}(u) \leq \phi(u)$ for $u \geq 0$, and $\hat{\phi}$ is convex on the interval $[0,1]$ (see $[8$, p. 255]).

Moreover, let us put

$$
\bar{\phi}(u)=\left(\phi^{*}\right)^{*}(u) \text { for } u \geq 0 .
$$

It is seen that $\bar{\phi}$ is a convex Orlicz function such that $\lim _{u \rightarrow \infty} \bar{\phi}(u) / u=\infty$. The relation between $\hat{\phi}$ and $\bar{\phi}$ is described by

LEMMA 2.2. We have $\hat{\phi} \sim \bar{\phi}$ and $\bar{\phi}(u) \leq \phi(u)$ for $u \geq 0$.

PROOF. First, we shall show that $\bar{\phi}(u) \leq \phi(u)$ for $u \geq 0$. Indeed, since $\lim _{v \rightarrow \infty} \phi^{*}(v) / v=\infty$, for every $u>0$ there exists $v_{u}>0$ such that $\bar{\phi}(u)+\phi^{*}\left(v_{u}\right)=u v_{u}$. But $u v_{u} \leq \phi(u)+\phi^{*}\left(v_{u}\right)$; hence $\bar{\phi}(u) \leq \phi(u)$ for $u \geq 0$. In [18, Lemma 2.1] it is proved that $\hat{\phi} \sim \bar{\phi}$ whenever $\liminf _{u \rightarrow 0} \phi(u) / u=0$. Now assume that $\liminf _{u \rightarrow 0} \phi(u) / u>0$. We can check that $\hat{\phi} \sim \chi_{1}$, where $\chi_{1}(u)=u$ for $u \geq 0$ (see [18]). It suffices to show that $\bar{\phi} \sim \chi_{1}$. In view of Lemma 2.1 there exists a number $v_{0}>0$ such that $\phi^{*}(v)=0$ for $0 \leq v \leq v_{0}$, and $\phi^{*}(v) \geq 0$ for $v>v_{0}$. Moreover, since $\lim _{v \rightarrow \infty} \phi^{*}(v) / v=\infty$, for every $u>0$ there exists $v_{u}>v_{0}$ such that $u v-\phi^{*}(v)<0$ for $v>v_{u}$. Hence, for every $u>0, \bar{\phi}(u)=\max \left(u v_{0}, \sup \left\{u v-\phi^{*}(v): v_{0} \leq v \leq v_{u}\right\}\right)$. But $\sup \left\{u v-\phi^{*}(\mathrm{v}): \mathrm{v}_{0} \leq \mathrm{v} \leq \mathrm{v}_{u}\right\}=u \mathrm{v}^{\prime}-\phi^{*}\left(\mathrm{v}^{\prime}\right)$ for some $\mathrm{v}^{\prime}$ with $\mathrm{v}_{0} \leq \mathrm{v}^{\prime} \leq \mathrm{v}_{u^{\prime}}$. Assuming that $\mathrm{v}_{0}<\mathrm{v}^{\prime}$, we obtain that $\bar{\phi}(u)=u \mathrm{v}_{0}$ for $0 \leq u \leq u_{0}=\phi^{*}\left(\mathrm{v}^{\prime}\right) /\left(\mathrm{v}^{\prime}-\mathrm{v}_{0}\right)$, and thus $\bar{\phi} \sim \chi_{1}$. 
For a topological vector space $(E, \xi)$ we shall denote by $(E, \xi)^{*}$ its topological dual. We shall denote by $\left(1^{\star}\right)^{*}$ the dual space of $\left(1^{\star}, \tau_{\phi}\right)$.

Let us recall that the Mackey topology of $(E, \xi)$ is the finest locally convex topology $\tau$ which produces the same continuous linear functionals as the original topology $\xi$. If $(E, \xi)$ is an $F$-space then $\tau$ is the finest locally convex topology on $E$ which is weaker than $\xi$ (see [24]).

Kalton [8] has showed that the Mackey topology $\tau_{\phi}$ of a separable Orlicz sequence space $1^{\star}$ coincides with the topology $\tau_{\left.\dot{\phi}\right|_{1}}$ induced from $1^{\phi}$. For an arbitrary $1^{\star}$, the Mackey topology $\tau_{\phi}$, has been described by Drewnowski and Nawrocki [5].

Denote by $\tau_{\phi}$ the Mackey topology of $\left(1^{\dagger}, \tau_{\phi}\right)$, by $\tau_{h^{\star}}$ the Mackey topology of $\left(h^{\dagger}, \tau_{\phi} h^{\dagger}\right)$, and by $\pi_{\phi}$ the topology defined by the Riesz seminorm $p_{\downarrow}$.

Combining [5, Theorems 5.1 and 5.3] with Lemma 2.2 we get the following important descriptions of $\tau_{h^{*}}$ and $\tau_{\phi}$.

THEOREM 2.3. The following equalities hold:

$$
\tau_{h^{\dagger}}=\tau_{\bar{\phi} \mid h^{*}}, \tau_{\phi}=\left(\tau_{\bar{\phi} \mid 1^{\dagger}}\right) \vee \pi_{\phi} .
$$

It is well-known (see [11], [12]) that the $F$-norm topology $\tau_{\bar{\phi}}$ on $1^{\bar{\phi}}$ can be generated by two Riesz norms:

$$
\begin{aligned}
\|x\|_{\bar{\phi}} & =\inf _{\lambda>0}\left\{\frac{1}{\lambda}\left(\rho_{\bar{\phi}}(\lambda x)+1\right)\right\} \\
& =\sup \left\{\left|\sum_{i=1}^{\infty} x(i) z(i)\right|: z \in 1^{\phi^{*}}, \rho_{\phi^{*}}(z) \leq 1\right\}
\end{aligned}
$$

and

$$
\|x\|_{\bar{\phi}}=\inf \left\{\lambda>0: \rho_{-}^{-}(x / \lambda) \leq 1\right\} .
$$

Moreover, $\|x\|_{\bar{\phi}} \leq\|x\|_{\bar{\phi}} \leq 2\|x\|_{\bar{\phi}}$ for all $x \in 1^{\bar{\phi}}$ and $\|x\|_{\bar{\phi}} \leq 1$ iff $\rho_{\bar{\phi}}(x) \leq 1$.

Therefore, in view of Theorem 2.3 the Mackey topology $\tau_{\phi}$ can be generated by two Riesz norms:

$$
p_{\phi} v\|\cdot\|_{\bar{\phi}} \text { and } p_{\phi} v\|\cdot\|_{\bar{\phi}}
$$

which will be of importance in our discussion. Thus two corresponding Riesz norms on $\left(1^{\dagger}\right)^{*}$ can be given by

$$
\begin{gathered}
\|f\|_{\phi}^{*}=\sup \left\{|f(x)|: x \in 1^{\phi}, \quad p_{\phi}(x) \leq 1 \quad \text { and }\|x\|_{\phi} \leq 1\right\} \\
\|f\|_{\phi}^{*}=\sup \left\{|f(x)|: x \in 1^{\phi}, \quad p_{\phi}(x) \leq 1 \text { and }\|x\|_{\bar{\phi}} \leq 1\right\} .
\end{gathered}
$$

Thus $\left(1^{\dagger}\right)^{*}$ is a Banach lattice under each of the norms $\|\cdot\|_{\phi}^{*}$ and $\|\cdot\|_{\phi}^{*}$. Moreover, since $\rho_{\phi}(x) \leq 1$ implies $p_{\phi}(x) \leq 1$ and $\rho_{\bar{\phi}}(x) \leq 1$, we can put (see [19]):

$$
\|f\|_{\rho_{\phi}}^{*}=\sup \left\{|f(x)|: x \in 1^{\dagger}, \quad \rho_{\phi}(x) \leq 1\right\} .
$$

We shall denote by $\left(1^{\phi}\right)^{-}$the collection of all order bounded linear functionals on $1^{\star}$. It is well-known that $\left(1^{\dagger}\right)^{-}=\left(1^{\dagger}\right)^{*}$ (see [1, Theorem 16.9]). An order bounded linear functional $f$ on $1^{\phi}$ is said to be order continuous (resp. singular) if $x_{\alpha} \stackrel{0}{\rightarrow} 0$ in $1^{\phi}$ implies $f\left(x_{\alpha}\right) \rightarrow 0$ for a net $\left(x_{\alpha}\right)$ in $1^{\phi}\left(\right.$ resp. $f(x)=0$ for all $\left.x \in h^{\phi}\right)$ (see $[9, \mathrm{Ch} . \mathrm{X}])$. The set of all order continuous (resp. singular) functionals on $1^{\dagger}$ will be denoted by $\left(1^{\phi}\right)_{n}$ (resp. $\left.\left(1^{\dagger}\right)_{s}^{\sim}\right)$.

The next theorem gives a characterization of the space $\left(1^{\star}\right)^{\circ}$.

THEOREM 2.4. (a) For a linear functional $f$ on $1^{\dagger}$ the following statements are equivalent: 
(1) $f$ is order bounded.

(2) $f$ is $\tau_{\phi}$-continuous.

(3) There exist unique $f_{n} \in\left(1^{\phi}\right)_{n}^{-}$and $f_{s} \in\left(1^{\phi}\right)_{s}^{-}$such that

$$
f(x)=f_{n}(x)+f_{s}(x) \text { for } x \in 1^{4} \text {. }
$$

(b) $\left(1^{\phi}\right)_{s}^{-}=\left(\left(1^{\dagger}\right)_{n}^{-d}\left(=\right.\right.$ the disjoint complement of $\left(1^{\phi}\right)_{n}^{-}$in $\left.\left(1^{\dagger}\right)^{*}\right)$, and moreover, $\left(1^{\dagger}\right)_{n}^{-}$and $\left(1^{\phi}\right)_{s}^{-}$are Banach lattices under each of the norms $\|\cdot\|_{\phi}^{*},\|\cdot\|_{\phi}^{*}$.

PROOF. (a) Since $\left(1^{\star}, p_{\downarrow} \vee\|\cdot\|_{\bar{\phi}}\right)^{*}=\left(1^{\dagger}\right)^{*}=\left(1^{\dagger}\right)^{\sim}$, by $\left[9, \mathrm{Ch}\right.$. VI, $\$ 1$, Theorem 5], we obtain that $\left(1^{\dagger}\right)_{n}^{-}$ separates the points of $1^{\star}$, and to get our result it suffices to use Theorem 6 of $[9, \mathrm{Ch} . \mathrm{X}, \S 3]$.

(b) Since $\left(1^{\dagger}\right)_{n}^{-}$is a band of $\left(1^{\dagger}\right)^{-}\left(\right.$see $\left[1\right.$, Theorem 3.7]) $\left(1^{\dagger}\right)_{n}^{-}$is a $\|\cdot\|_{\phi}^{*}$-closed (resp. $\|\cdot\| \|_{\phi}^{\infty}$-closed) subspace of $\left(1^{\dagger}\right)^{*}$ (see [1, Theorem 5.6]). Thus $\left(1^{\dagger}\right)_{n}^{-}$is a Banach lattice, because $\left(1^{\star}\right)^{*}$ is a Banach lattice. Moreover, since $\left(1^{\dagger}\right)_{s}^{-}=\left(\left(1^{\phi}\right)_{n}^{-}\right)^{d},\left(1^{\dagger}\right)_{s}^{\sim}$ is a band of $\left(1^{\dagger}\right)^{-}\left(\right.$see $[1$, p. 27] $)$, and by the above argument $\left(1^{\downarrow}\right)_{s}^{-}$ is a Banach lattice.

In view of [17] the conjugate $\bar{\rho}_{\phi}$ of the modular $\rho_{\phi}$ can be defined on the algebraic dual $\overline{1}^{\star}$ of $1^{\star}$ as follows:

$$
\overline{\rho_{\phi}}(f)=\sup \left\{|f(x)|-\rho_{\phi}(x): x \in 1^{\star}\right\} .
$$

Note that if $f \geq 0$, then

$$
\bar{\rho}_{\phi}(f)=\sup \left\{f(x)-\rho_{\phi}(x): 0 \leq x \in \omega, \rho_{\phi}(x)<\infty\right\} .
$$

Indeed, since $|f(x)| \leq f(|x|)$ (see [1, p. 21]) and $\rho_{\phi}(x)=\rho_{\phi}(|x|)$ we have

$$
\begin{aligned}
\bar{\rho}_{\phi}(f) & \left.\leq \sup f(|x|)-\rho_{\phi}(|x|): \rho_{\phi}(|x|)<\infty\right\} \\
& \leq \sup \left\{f(x)-\rho_{\phi}(x): 0 \leq x \in \omega, \rho_{\phi}(x)<\infty\right\} .
\end{aligned}
$$

We shall need the following definition.

A linear functional $f$ on $1^{t}$ is said to be bounded for $\rho_{\omega}$ (see [16], [17]) if there exists $\gamma>0$ such that

$$
|f(x)| \leq \gamma\left(\rho_{\phi}(x)+1\right) \text { for } x \in 1^{\star} \text {. }
$$

The collection of all bounded for $\rho_{\phi}$ linear functionals on $1^{t}$ will be denoted by $\overline{1^{\phi}}$.

The basic properties of $\bar{\rho}_{q}$ are included in the following

THEOREM 2.5. The conjugate $\bar{\rho}_{\phi}$ of the modular $\rho_{\phi}$ is a convex orthogonal additive modular on $\overline{1^{\dagger}}$. Moreover, the following equality holds: $\left(1^{\star}\right)^{*}=\overline{1^{\dagger}}$.

Proof. Using $[17, \S 4]$ and arguing as in the proof of $\left[16\right.$, Theorem 38.2] we obtain that $\bar{\rho}_{\phi}$ is a convex orthogonal additive modular on $\overline{1^{\phi}}$. To end the proof it suffices to show that $\left(1^{\phi}\right)^{\bullet}=\overline{1^{\phi}}$. Indeed, let $f \in\left(1^{\phi}\right)^{*}$ and $\rho_{\phi}(x)<\infty$. Then $p_{\phi}(x) \leq 1$ and there exists $\gamma>0$ such that $|f(x)| \leq \gamma\left(\max \left(p_{\phi}(x),\|x\|_{\bar{\phi}}\right)\right) \leq \gamma\left(\rho_{\phi}(x)+1\right)$ $\leq \gamma\left(\rho_{\phi}(x)+1\right)$, because $\bar{\phi}(u) \leq \phi(u)$ for $u \geq 0$. Thus $f \in \overline{1^{\natural}}$; hence $\left(1^{\dagger}\right)^{*} \subset \overline{1^{\dagger}}$. Next, let $f \in \overline{1^{\dagger}}$ and let $|x|_{\downarrow}<1$. Then $\rho_{\phi}(x) \leq 1$, and hence $|f(x)| \leq 2 \gamma$ for some $\gamma>0$. This means that $f \in\left(1^{\phi}\right)^{*}$, and thus $\overline{1^{*}} \subset\left(1^{\star}\right)^{*}$. The proof is completed.

Thus by means of $\bar{\rho}_{\phi}$ two modular norms can be defined on $\left(1^{\dagger}\right)^{*}$ in a usual way (see [16], [17]):

$$
\|f\|_{\bar{\rho}_{\phi}}=\inf _{\lambda>0}\left\{\frac{1}{\lambda}\left(\bar{\rho}_{\phi}(\lambda f)+1\right)\right\} \quad \text { (the first modular norm) }
$$




$$
\|f\|_{\bar{\rho}_{\phi}}=\inf \left\{\lambda>0: \bar{\rho}_{\phi}(f / \lambda) \leq 1\right\} \quad \text { (the second modular norm). }
$$

3. Order Continuous Linear Functionals on $1^{\dagger}$. We shall start this section with a description of the Köthe dual $\left(1^{\natural}\right)^{x}$ of $1^{t}$ that will be useful in obtaining a corresponding characterization of order continuous linear functional on $1^{\dagger}$ (see [20, Proposition 1.9]).

Let us recall that the Köthe dual $S^{x}$ of a sequence space $S$ is the sequence space defined by (see [10, \$30.1]):

$$
S^{x}=\left\{y \in \omega: \sum_{i=1}^{\infty}|x(i) y(i)|<\infty \text { for all } x \in S\right\} .
$$

THEOREM 3.1. The following equalities hold:

$$
\left(1^{\dagger}\right)^{x}=\left(h^{\dagger}\right)^{x}=\left(h^{\bar{\phi}}\right)^{x}=1^{\dagger} .
$$

In particular, if $\liminf _{u \rightarrow 0} \phi(u) / u>0$, then $\left(1^{\dagger}\right)^{*}=1^{\infty}$.

PROOF. First, we shall show that $\left(1^{\phi}\right)^{x}=\left(h^{\phi}\right)^{x}=\left(h^{\phi}\right)^{x}$. Since $\left(1^{\phi}\right)^{x} \subset\left(h^{\phi}\right)^{x}$ and $\left(h^{\bar{\gamma}}\right)^{x} \subset\left(h^{\phi}\right)^{x}$, it suffices to show that $\left(h^{\dagger}\right)^{x} \subset\left(1^{\dagger}\right)^{x}$ and $\left(h^{\phi}\right)^{x} \subset\left(h^{\phi}\right)^{x}$. Indeed, let $y \in\left(h^{\dagger}\right)^{x}$, i.e., $\sum_{i=1}^{\infty}|z(i) y(i)|<\infty$ for all $z \in h^{\phi}$. Putting

$$
g_{y}(z)=\sum_{i=1}^{\infty} z(i) y(i) \text { for } z \in h^{\dagger} \text {, }
$$

by [20, Proposition 1.9] and Theorem 2.3 we get

$$
g_{y} \in\left(h^{\dagger}\right)_{n}^{-}=\left(h^{\dagger}\right)^{-}=\left(h^{\dagger}, \tau_{\phi \mid h^{\dagger}}\right)^{*}-\left(h^{\dagger}, \tau_{\phi \mid h^{\dagger}}\right)^{*} \text {. }
$$

Therefore, we can put

$$
\left\|g_{y}\right\|_{\bar{\phi}}=\sup \left\{\left|\sum_{i=1}^{\infty} z(i) y(i)\right|: z \in h^{\dagger}, \quad\|z\|_{\bar{\phi}} \leq 1\right\} .
$$

Let now $x \in 1^{\dagger}\left(\right.$ resp. $\left.x \in h^{\bar{\gamma}}\right), x \neq 0$. We shall show that $\sum_{i=1}^{\infty}|x(i) y(i)|<\infty$. Since $x \in 1^{\bar{\phi}}$ and $x^{(n)} \in h^{\dagger}$ we get

$$
\begin{aligned}
& \frac{1}{\|x\|_{\bar{\phi}}} \sum_{i=1}^{\infty}|x(i) y(i)|=\frac{1}{\|x\|_{\bar{\phi}}} \sup _{n} \sum_{i=1}^{\infty}\left|x^{(n)}(i)\right| \cdot \operatorname{sign} y(i) \cdot y(i) \\
& \quad \leq \sup \left\{\left|\sum_{i=1}^{\infty} z(i) y(i)\right|: z \in h^{\phi}, \quad\|z\|_{\bar{\phi}} \leq 1\right\}-\left\|g_{y}\right\|_{\bar{\delta}}<\infty .
\end{aligned}
$$

Hence $y \in\left(1^{\phi}\right)^{x}$ (resp. $\left.y \in\left(h^{\bar{\phi}}\right)^{x}\right)$, so that $\left(1^{\phi}\right)^{x}=\left(h^{6}\right)^{x}=\left(h^{\bar{\phi}}\right)^{x}$.

We have $\left(h^{\bar{\gamma}}\right)_{n}^{-}=\left(h^{\bar{\phi}}\right)^{-}=\left(h^{\bar{\phi}}, \tau_{\bar{\phi} \mid h^{\bar{\gamma}}}\right)^{*}$. It is well-known that by the mapping $\left(y \rightarrow g_{y}\right)$ the space $\left(h^{\bar{\phi}}\right)^{x}$ can be identified with $\left(h^{\bar{\phi}}\right)_{n}^{-}$(see [20, Proposition 1.9]), and the space $1^{\vec{\phi}}$ with $\left(h^{\bar{\phi}}, \tau_{\bar{\phi} \mid h^{\bar{\phi}}}\right)$ (see [12, Ch. II, \$3, Theorem 2]). Thus $\left(h^{\bar{\phi}}\right)^{x}=1^{\bar{\phi}}$, and since $\bar{\phi}^{*}-\phi^{\cdots * *}=\phi^{*}$, the proof is complete.

REMARK. The equality $\left(1^{\dagger}\right)^{x}=1^{t}$ has been obtained by the author in [18] in a different way, using the so-called modular topology on $1^{\natural}$.

REMARK. Assume now that $\phi$ is an Orlicz function, not necessarily satisfying the condition: $\phi(u) / u \rightarrow \infty$ as $u \rightarrow \infty$. Let $\psi$ be any Orlicz function such that $\psi(u)-\phi(u)$ for $0 \leq u \leq 1$, and $\psi(u) / u \rightarrow \infty$ as $u \rightarrow \infty$. Then in view of Theorem 3.1 we get $\left(1^{\dagger}\right)^{x}=\left(1^{\psi}\right)^{x}=1^{\psi}$. Thus, by Lemma 3.1 we get $\left(1^{p}\right)^{x}=1^{\infty}$ for $0<p \leq 1$.

We are now able to give a characterization of order continuous linear functionals on $1^{\phi}$.

THEOREM 3.2. Let $f$ be a linear functional on $1^{\phi}$.

(a) The following statements are equivalent: 
(1) $f$ is order continuous.

(2) There exists a unique $y \in 1^{\dagger}$ such

$$
f(x)=f_{y}(x)=\sum_{i=1}^{\infty} x(i) y(i) \quad \text { for all } x \in 1^{4} .
$$

(b) If $f$ is order continuous, then the following equalities hold:

$$
\begin{aligned}
\bar{\rho}_{\phi}(f) & =\rho_{\phi} \cdot(y), \\
\|f\|_{\phi}^{*} & =\|f\|_{\bar{\rho}_{\phi}}=\|y\|_{\phi}, \\
\|f\|_{\phi}^{*} & =\|f\|_{\bar{p}_{\phi}}=\|y\|_{\phi} .
\end{aligned}
$$

(c) Moreover, the map $1^{*} \supset y \rightarrow f_{y} \in\left(1^{\dagger}\right)_{n}^{-}$is a Riesz isomorphism.

PROOF. (a) It follows from [20, Proposition 1.9] and Theorem 3.1.

(b) By (a) we have $f(x)=\sum_{i=1}^{\infty} x(i) y(i)$ for some $y \in 1^{\circ}$ and all $x \in 1^{*}$.

First, we shall show that $\bar{\rho}_{\phi}(f)=\rho_{\phi} \cdot(y)$. From the definition of $\phi^{*}$ we easily obtain that $\bar{\rho}_{\phi}(f) \leq \rho_{\phi} \cdot(y)$. To prove that $\bar{\rho}_{\downarrow}(f) \geq \rho_{\downarrow} \cdot(y)$ let us note that there exists $0 \leq z \in \omega$ such that

$$
\phi(z(i))+\phi^{*}(|y(i)|)=|z(i) y(i)| \text { for } i=1,2, \ldots
$$

Putting $x(i)=(\operatorname{sign} y(i)) \cdot z(i)$ for $i=1,2, \ldots$, we get

$$
\begin{aligned}
\rho_{\phi} \cdot(y) & =\sum_{i=1}^{\infty} \dot{\phi}^{\dot{ }}(|y(i)|) \\
& =\sup _{n}\left\{\sum_{i=1}^{n}|z(i) y(i)|-\sum_{i=1}^{n} \phi(z(i))\right\} \\
& \leq \sup _{n}\left\{\left|\sum_{i=1}^{\infty} x^{(n)}(i) y(i)\right|-\sum_{i=1}^{\infty} \phi\left(\left|x^{(n)}(i)\right|\right)\right\} \leq \bar{\rho}_{\phi}(f) .
\end{aligned}
$$

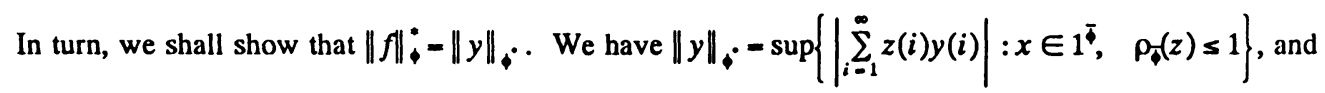
hence $\|f\|_{i}^{*} \leq\|y\|_{\ddagger}$. On the other hand, let $z \in 1^{\bar{\phi}}$ with $\rho_{-(}(z) \leq 1$. Putting $x(i)=(\operatorname{sign} y(i)) \cdot|z(i)|$ $(i=1,2, \ldots)$, we have $p_{\phi}\left(x^{(n)}\right)=0$ and $\rho_{-}\left(x^{(n)}\right) \leq \rho_{-}^{-}(z) \leq 1$. Thus

$$
\begin{aligned}
\left|\sum_{i=1}^{\infty} z(i) y(i)\right| & \leq \sup _{n} \sum_{i=1}^{\infty}\left|z^{(n)}(i) y(i)\right| \\
& =\sup _{n}\left|\sum_{i=1}^{\infty} x^{(n)}(i) y(i)\right| \leq\|f\|^{*} .
\end{aligned}
$$

Thus $\|y\|_{\phi} \leq\|f\|_{\phi}^{*}$ and hence $\|f\|_{+}^{*}=\|y\|_{\odot}$.

Moreover, since $\bar{\rho}_{\phi}(\lambda f)=\rho_{\phi} \cdot(\lambda y)$ for $\lambda>0$, we get $\|f\|_{\bar{\rho}_{\phi}}=\|y\|_{\phi}$.

Next, we shall show that $\|f\|_{\phi}^{*} \leq\|y\|_{\phi}$. To prove that $\|f\|_{\phi}^{*} \leq\|y\|_{\phi}$, let us assume that $x \in 1^{\phi}, p_{\phi}(x) \leq 1$ and $\|x\|_{\bar{\phi}} \leq 1$. Then $x \in 1^{\bar{\phi}}$, and by the Hölder's inequality (see $[11, \S 9]$ ) we get $|f(x)| \leq\|x\|_{\phi^{-}} \cdot\|y\|\left\|_{\phi} \leq \mid\right\| y \|_{\phi^{*}}$, because $\bar{\phi}^{*}=\bar{\phi}$. Thus $\|f\|_{\phi}^{*} \leq\|y\|_{\phi}$. To prove that $\|y\|\left\|_{\phi} \leq\right\| f \|_{\phi}^{*}$ let us note that (see [11, p. 135]): 


$$
\|y\|_{i}=\sup \left\{\left|\sum_{i=1}^{\infty} z(i) y(i)\right|: z \in 1^{\bar{\phi}},\|z\|_{\bar{\phi}} \leq 1\right\} .
$$

Let now $z \in 1^{\bar{\phi}}$ and $\|z\|_{\bar{\zeta}} \leq 1$. Putting $x(i)=(\operatorname{sign} y(i)) \cdot|z(i)|(I=1,2, \ldots)$ we have $p_{\phi}\left(x^{(n)}\right)=0$, $\|x(n)\|_{\bar{\phi}} \leq\|z\|_{\bar{\phi}} \leq 1$, and as above we get $\|y\|_{\phi} \leq\|f\|_{\phi}$.

Finally, since $\bar{\rho}_{\phi}(f / \lambda)=\rho_{\phi}(y / \lambda)$ for $\lambda>0$, we get $\|f\|_{\bar{\rho}_{\phi}}-\|y\|_{\phi}$.

(c) See $[9, \mathrm{Ch} . \mathrm{VI}, \S 1$, Theorem 1] and [14, Theorem 18.5].

REMARK. The general form of $\phi$-continuous (continuous with respect to the modular $\rho_{\phi}$ ) linear functionals on an Orlicz space $L^{\phi}(a, b)$ defined by an Orlicz function satisfying conditions $\phi(u) / u \rightarrow 0$ as $u \rightarrow 0$ and $\phi(u) / u \rightarrow \infty$ as $u \rightarrow \infty$, has been found by W. Orlicz [19].

4. Singular Linear Functionals on $1^{\dagger}$. In this section we assume that $\phi$ does not satisfy the $\Delta_{2}$-condition at 0 , because otherwise $\left(1^{\dagger}\right)_{s}^{-}=\{0\}$.

The following lemma describes positive singular linear functionals on $1^{\star}$.

LEMMA 4.1. Let $f$ be a positive singular linear functional on $1^{\star}$.

(a) For any $\varepsilon>0$ there exists $0 \leq y \in \omega$ with $\rho_{\varangle}(y)<\varepsilon$ such that $\|f\|_{\phi}^{*} \leq f(y)$.

(b) The following equalities hold:

$$
\begin{aligned}
\rho_{\phi}(f) & =\|f\|_{\rho_{\phi}}^{*}-\|f\|_{\phi}^{*}-\|f\|_{\phi}^{*} \\
& =\sup \left\{f(x): 0 \leq x \in \omega, \quad \rho_{\phi}(x)<\infty\right\} .
\end{aligned}
$$

(c) There exists $0 \leq y \in \omega$ with $\rho_{\triangleleft}(y)<\infty$ such that

$$
\left\|f_{A}\right\|_{;}^{*}=f\left(y_{A}\right) \text { for any subset } A \text { of } N
$$

and

$$
p_{\phi}\left(y_{A}\right)=1 \text { for any subset } A \text { of } N \text { with }\left\|f_{A}\right\|_{\phi}^{*} \neq 0 \text {. }
$$

PROOF. (a) Let $\varepsilon>0$ be given. Since (see [26, Lemma 102.1])

$$
\|f\|_{\dagger}^{*}=\sup \left\{f(x): 0 \leq x \in 1^{\dagger}, \quad p_{\phi}(x) \leq 1, \quad \rho_{-(x)}^{-}(x),\right.
$$

for every $k \in N$ there exists $0 \leq z_{k} \in 1^{\phi}$ such that $p_{\phi}\left(z_{k}\right)<1$ and $\|f\|_{\phi}^{*} \leq f\left(z_{k}\right)+\frac{1}{k}$. Then $\rho_{\phi}\left(z_{k}\right)<\infty$ and there exists a strictly increasing sequence of natural numbers $\left(n_{k}\right)$ such that

$$
\rho_{\downarrow}\left(z_{k}-z_{k}^{\left(n_{k}\right)}\right)=\sum_{i=n_{k}}^{\infty} \phi\left(z_{k}(i)\right)<\frac{\varepsilon}{2^{k}} .
$$

Let $x_{k}=z_{k}-z_{k}^{\left(w_{k}\right)}$ for $k=1,2, \ldots$. Then in view of the axion (C) of completeness of the modular $\rho_{q}$ there exists $0 \leq y \in \omega$ such that $x_{k} \leq y$, for all $k \in N$, and $\rho_{\phi}(y) \leq \sum_{k=1}^{\infty} \rho_{\phi}\left(x_{k}\right)<\varepsilon$. But $z_{k}^{\left(n_{k}\right)} \in h^{\downarrow}$ for all $k \in N$, so that

$$
\begin{aligned}
\|f\|_{\phi}^{*} & \leq f\left(z_{k}=z_{k}^{\left(n_{k}\right)}\right)+f\left(z_{k}^{\left(n_{k}\right)}\right)+\frac{1}{k} \\
& =f\left(x_{k}\right)+\frac{1}{k} \leq f(y)+\frac{1}{k} .
\end{aligned}
$$

Since $\varepsilon>0$ and $k$ are arbitrary, we conclude that $\|f\|_{i}^{*} \leq f(y)$.

(b) We have

$$
\|f\|_{\phi}^{*} \leq\|f\|_{\phi}^{*} \leq \sup \left\{f(x): 0 \leq x \in 1^{\dagger}, \quad p_{\phi}(x) \leq 1, \quad \rho_{\phi}(x)<\infty\right\} .
$$

To prove that $\sup \left\{f(x): 0 \leq x \in 1^{\dagger}, \quad p_{\phi}(x) \leq 1, \quad \rho_{-}^{-}(x)<\infty \leq\|f\|_{\phi}^{*}\right.$ assume that $0 \leq x \in 1^{\dagger}$ and 
$p_{\phi}(x) \leq 1, \quad \rho_{\phi}(x)<\infty$. Given an $\eta>0$, there exists $n \in N$ such that $\rho_{\bar{\phi}}\left(x-x^{(n)}\right)<\eta$. Then

$$
\left\|x-x^{(n)}\right\|_{\bar{\phi}} \leq 1+\rho_{\phi}\left(x-x^{(n)}\right) \leq 1+\eta
$$

and

$$
\begin{aligned}
f(x) & =f\left(x-x^{(n)}\right)+f\left(x^{(n)}\right)=f\left(x-x^{(n)}\right) \\
& \leq(1+\eta)\|f\|_{\varnothing}^{*} .
\end{aligned}
$$

Hence $f(x) \leq\|f\|_{\phi}^{\bullet}$, and thus we obtain

$$
\|f \mid\|=\|f\|_{\phi}^{*}=\sup \left\{f(x): x \in 1^{\dagger}, \quad p_{\phi}(x) \leq 1, \quad \rho_{\phi}(x)<\infty\right\} .
$$

Moreover, by (a) there exists $0 \leq y \in \omega$, with $\rho_{\downarrow}(y) \leq 1$, such that $\|f\|_{\phi}^{*} \leq f(y)$. Hence

$$
\begin{aligned}
\|f\|_{\rho_{\phi}}^{*} & =\sup \left\{f(x): 0 \leq x \in \omega, \quad \rho_{\phi}(x) \leq 1\right\} \\
& \leq \sup \left\{f(x): 0 \leq x \in \omega, \quad \rho_{\phi}(x)<\infty\right\} \\
& \leq \sup \left\{f(x): x \in 1^{\dagger}, \quad p_{\phi}(x) \leq 1, \quad \rho_{\phi}(x)<\infty\right\} \\
& =\|f\|_{\phi}^{*} \leq f(y) \leq \sup \left\{f(x): 0 \leq x \in \omega, \quad \rho_{\phi}(x) \leq 1\right\} .
\end{aligned}
$$

Thus we proved that

$$
\|f\|_{\rho_{\phi}}^{*}=\|f\|_{\phi}^{*}=\|f\|_{\phi}^{*}=\sup \left\{f(x): 0 \leq x \in \omega, \rho_{\phi}(x)<\infty\right\} .
$$

Finally, we shall show that $\bar{\rho}_{\phi}(f)=\|f\|_{\phi}^{*}$. Indeed, by (a), for every $n \in N$, there exists $0 \leq y_{n} \in \omega$, with $\rho_{\phi}\left(y_{n}\right) \leq \frac{1}{n}$, and such that $\|f\|_{\phi}^{*} \leq f\left(y_{n}\right)$. Hence

$$
\begin{aligned}
\bar{\rho}_{\phi}(f) & =\sup \left\{f(x)-\rho_{\phi}(x): 0 \leq x \in \omega, \quad \rho_{\phi}(x)<\infty\right\} \\
& \geq f\left(y_{n}\right)-\rho_{\phi}\left(y_{n}\right) \geq\|f\|_{\phi}^{*}-\frac{1}{n} .
\end{aligned}
$$

Hence $\bar{\rho}_{\phi}(f) \geq\|f\|_{\phi}^{*}$, and since

$$
\bar{\rho}_{q}(f) \leq \sup \left\{f(x): 0 \leq x \in \omega, \quad \rho_{\phi}(x)<\infty\right\}=\|f\|
$$

we get $\bar{\rho}_{\phi}(f)=\|f\|_{\phi}$. Thus the proof of (b) is completed.

(c) Let $A$ be a subset of $\mathrm{N}$, and let $0 \leq x \in \omega$ with $\rho_{\phi}(x)<\infty$ be given. Arguing as in (a) we obtain that there exists $0 \leq z_{k} \in \omega$ with $\rho_{\phi}\left(z_{k}\right)<\infty(k=1,2, \ldots)$ such that $\|f\|_{i} \leq f\left(z_{k}\right)+\frac{1}{k}$. Since $\|f\|_{\phi}^{*}=\sup \left\{f(z): 0 \leq z \in \omega, \rho_{\phi}(z)<\infty\right\}$ (see (b)), we have

$$
f\left(x \vee z_{k}\right) \leq f\left(z_{k}\right)+\frac{1}{k} .
$$

for all $k \in N$, because $\rho_{\phi}\left(x \vee z_{k}\right) \leq \rho_{\phi}(x)+\rho_{\phi}\left(z_{k}\right)<\infty$. But $\left(x \vee z_{k}-z_{k}\right)_{A} \leq x \vee z_{k}-z_{k}$, so we get

$$
f\left(x_{A}\right) \leq f\left(\left(x \vee z_{k}\right)_{A}\right) \leq f\left(\left(z_{k}\right)_{A}\right)+\frac{1}{k} \quad(k=1,2, \ldots) .
$$

Choose an increasing sequence of natural numbers $\left(m_{k}\right)$ such that $\rho_{\phi}\left(z_{k}-z^{\left(m_{k}\right)}\right)<\frac{1}{z^{k}}$, and let $x_{k}=z_{k}-z_{k}^{\left(m_{k}\right)}$. Then in view of the axiom (C) of completeness of $\rho_{\phi}$, there exists $0 \leq y \in \omega$ such that $x_{k} \leq y$ for all $k \in N$, and $\rho_{\phi}(y) \leq 1$. Hence

$$
\begin{aligned}
f\left(x_{A}\right) & \leq f\left(\left(z_{k}-z_{k}^{\left(m_{k}\right)}\right)_{A}\right)+f\left(\left(z_{k}^{\left(m_{k}\right)}\right)_{A}\right)+\frac{1}{k} \\
& =f\left(\left(x_{k}\right)_{A}\right)+\frac{1}{k} \leq f\left(y_{A}\right)+\frac{1}{k} .
\end{aligned}
$$


Thus we obtain that $\left\|f_{A}\right\|_{\leftarrow}^{*}=f\left(y_{A}\right)$, because by (b),

$$
\left\|f_{A}\right\|_{\downarrow}^{*}=\sup \left\{f\left(x_{A}\right): 0 \leq x \in \omega, \quad \rho_{\phi}(x)<\infty\right\} .
$$

Assume now that $\left\|f_{A}\right\|_{\phi}^{*} \neq 0$. Given $\eta>0$ we have $\rho_{\phi}\left(y_{A} /\left(p_{\phi}\left(y_{A}\right)+\eta\right)\right)<\infty$, and hence, by (b), $\left\|f_{A}\right\|_{\phi}^{*} \geq f\left(\left(y_{A}\left(\left(p_{\phi}\left(y_{A}\right)+\eta\right)\right)\right.\right.$. Thus $\left\|f_{A}\right\|_{\phi}^{*}-f\left(y_{A}\right) \leq\left(p_{\phi}\left(y_{A}\right)+\eta\right)\left\|f_{A}\right\|_{\phi}^{*}$, so $p_{\phi}\left(y_{A}\right)-1$, because $p_{\phi}\left(y_{A}\right) \leq p_{\phi}(y) \leq 1$. Thus the proof of $(c)$ is completed.

COROLLARY 4.2. The space $\left(\left(1^{+}\right)_{s}^{\sim},\left\|^{*}\right\|_{s}^{*}\right)$ is an abstract L-space.

PROOF. By Theorem 2.4, $\left(\left(1^{*}\right)_{s}^{*},\left\|^{*}\right\|^{*}\right)$ is a Banach lattice. Arguing as in the proof of Lemma 2 of [2] we can show that $\left\|f_{1}+f_{2}\right\|_{\phi}^{*}=\left\|f_{1}\right\|_{\phi}^{*}+\left\|f_{2}\right\|_{\phi}^{*}$ for any $f_{1}, f_{2} \in\left(\left(1^{\dagger}\right)_{s}^{-}\right)^{+}$, and this means that $\left(1^{*}\right)_{s}^{-}$is an abstract L-space (see [23, Ch. II, §9]).

By $b a(N)$ we denote the family of all bounded real valued finitely additive set functions on $\mathbf{N}$. It is known that $b a(N)$ is a vector lattice with the usual ordering: $v_{1} \geq v_{2}$ iff $v_{1}(A) \geq v_{2}(A)$ for all $A \subset N$. Then $v=v^{+}-v^{-}$and $|v|=v^{+}+v^{-}$, where $v^{+}$and $v^{-}$denote the positive and the negative part of $v \in b$ a(N). Moreover $b a(N)$ is a Banach space under the norm $\|v\|-|v|(N)$ (see [6, Ch. III, 1.4, 1.7]).

For given $f \in\left(\left(1^{\dagger}\right)_{s}^{-}\right)^{+}$let us put $v_{f}(A)-\left\|f_{A}\right\|_{\text {; }}^{*}$ for any subset $A$ of $\mathbf{N}$. Then by Corollary 4.2, $v_{f} \in(b a(N))^{+}$and $\left\|v_{f}\right\|=v_{f}(\mathbf{N})=\|f\|^{*}$.

The following definition is justified by Lemma 4.1.

$A v \in b a(N)$ is said to be in class $B_{\phi}(N)$ if there exists $0 \leq y \in \omega$, with $\rho_{\phi}(y)<\infty$, such that $p_{\phi}\left(y_{A}\right)=1$ for any subset $A$ of $N$ with $|v|(A) \neq 0$.

One can show that $B_{\phi}(N)$ is a Riesz subspace of $b a(N)$. In view of Lemma 4.1 we have the following

LEMMA 4.3. If $f \in\left(\left(1^{\dagger}\right)_{s}^{-}\right)^{+}$, then $v_{f} \in\left(B_{\phi}(\mathrm{N})\right)^{+}$.

Thus we can define a mapping $T:\left(\left(1^{+}\right)_{s}^{-}\right)^{+} \rightarrow\left(B_{\triangleleft}(N)\right)^{+}$given by

$$
T(f)=v_{f} \text { for any } f \in\left(\left(1^{+}\right)_{s}^{-}\right)^{+} \text {. }
$$

In view of Corollary 4.2 the mapping $T$ is additive.

For any $v \in(b a(N))^{+}$we define a positive functional $I_{v}$ on $\left(1^{\dagger}\right)^{+}$by

$$
I_{v}(x)=\inf \left\{\sum_{k=1}^{n} p_{\phi}\left(x_{A_{k}}\right) v\left(A_{k}\right)\right\}
$$

where the infimum is taken over all finite disjoint partitions $\left(A_{k}\right)_{1}^{\infty}$ of $\mathbf{N}$.

By the same argument as in the proof of Lemma 5 of [2] we can prove that the functional $I_{v}$ is additive on $\left(1^{\dagger}\right)^{+}$. Thus $I_{v}$ has a unique positive extension to a linear functional on $1^{\dagger}$ (see [1, Lemma 3.1]). This extension (denoted again by $I_{v}$ ) is given by $I_{v}(x)=I_{v}\left(x^{+}\right)-I_{v}\left(x^{-}\right)$for all $x \in 1^{+}$.

LEMMA 4.4. If $v \in(b a(N))^{+}$, then $I_{v} \in\left(\left(1^{*}\right)_{s}^{-}\right)^{+}$and $\left\|I_{v}\right\|_{*}^{*} \leq v(N)$.

PROOF. Since $I_{v}$ is positive on $I^{\phi}, I_{v}$ is order bounded. It is seen that $I_{v}(x)=0$ for all $x \in h^{\phi}$, so $I_{v} \in\left(\left(1^{\dagger}\right)_{s}^{-}\right)^{+}$. Moreover, $\left|I_{v}(x)\right| \leq I_{v}\left(x^{+}\right)+I_{v}\left(x^{-}\right)=I_{v}(|x|) \leq p_{\phi}(x) v(\mathrm{~N})$ for all $x \in 1^{\phi}$, so $\left\|I_{v}\right\|_{\phi}^{*} \leq v(\mathrm{~N})$. 
Thus we can define a mapping $G:\left(B_{\phi}(\mathrm{N})\right)^{+} \rightarrow\left(\left(1^{+}\right)_{s}^{-}\right)^{+}$by

$$
G(v)=I_{v} \text { for any } v \in\left(B_{\phi}(\mathrm{N})\right)^{+} \text {. }
$$

THEOREM 4.5. The following statements hold:

(1) $(G \circ T)(f)=f$ for any $f \in\left(\left(1^{\natural}\right)_{s}^{-}\right)^{+}$, i.e.,

$$
f(x)=I_{v f}(x) \text { for all } x \in 1^{\dagger} \text {. }
$$

(2) $(T \circ G)(v)=v$ for any $v \in(B(N))^{+}$, i.e.,

$$
v(A)=\left\|\left(I_{v}\right)_{A}\right\|_{\text {; }}^{*} \text { for any subset } A \text { of } \mathrm{N} \text {. }
$$

PROOF. (1) Using Corollary 4.2 and Lemma 4.4, it suffices to repeat the arguments of the proof of Theorem 2 of [2].

(2) We first prove the case $A=N$. Since $\nu \in\left(B_{\phi}(N)\right)^{+}$, there exists $0 \leq y \in \omega$ such that $\rho_{\phi}(y)<\infty$ and $p_{\phi}\left(y_{E}\right)=1$ for any subset $E$ of $N$ with $v(E)>0$. Then for any finite disjoint partition $\left(E_{k}\right)_{1}^{n}$ of $N$ we have $\sum_{k=1}^{n} p_{\phi}\left(y_{E_{k}}\right) v\left(E_{k}\right)=v(\mathrm{~N})$, so $I_{v}(y)=v(\mathrm{~N})$. According to Lemma 4.1, we have $\left\|I_{v}\right\|_{\phi}^{\bullet} \geq I_{v}(y)=v(\mathrm{~N})$. Moreover, we have $I_{v}(x) \leq p_{\phi}(x) v(\mathrm{~N})$ for all $0 \leq x \in 1^{\natural}$. Hence $\left\|I_{v}\right\|_{\phi}^{*} \leq v(\mathrm{~N})$, so $\left\|I_{v}\right\|_{\phi}^{*}=v(\mathrm{~N})$. Assume now that $A$ is a fixed subset of $N$, and let $v_{1}(B)=v(A \cap B)$ for any $B \subset N$. One can easily show that $I_{v_{1}}=\left(I_{v}\right)_{A}$. Hence, by the above, we get $\left\|\left(I_{v}\right)_{A}\right\|_{\phi}^{*}=\left\|I_{v_{1}}\right\|_{\phi}^{*}=v_{1}(N)=v(\Lambda)$, and the proof is completed.

By Theorem 4.5 the mapping $G$ is additive, because $T$ is additive. Thus $T$ and $G$ have unique positive extensions to linear mappings $\tilde{T}:\left(1^{\dagger}\right)_{s}^{-} \rightarrow B_{\phi}(\mathrm{N})$ and $\tilde{G}: B_{q}(\mathrm{~N}) \rightarrow\left(1^{\dagger}\right)_{s}^{-}$(see [1, Lemma 3.1]) given by

$$
\tilde{T}(f)=v_{f^{+}}-v_{f^{-}} \text {and } \tilde{G}(v)=I_{v^{+}}-I_{v}
$$

Let us put: $v_{f}=v_{f^{+}}-v_{f^{-}}$and $I_{v}=I_{v^{*}}-I_{v^{-}}$. For any $v \in B_{q}(\mathrm{~N})$ we shall write

$$
\int x d v=I_{v}(x) \text { for all } x \in 1^{\natural} \text {. }
$$

THEOREM 4.6. (see [2, Theorem 4]). The mapping $\tilde{T}:\left(1^{\dagger}\right)_{s}^{-} \rightarrow B_{\uparrow}(\mathrm{N})$ is a Riesz isomorphism.

PROOF. In view of Theorem 4.5, we get $(\tilde{G} \circ \tilde{T})(f)-f$, for any $f \in\left(1^{\dagger}\right)_{s}^{-}$, and $(\tilde{T} \circ \tilde{G})(v)-v$, for any $v \in B_{\downarrow}(\mathbf{N})$. Thus $\tilde{T}$ is a Riesz isomorphism, because $T$ is positive (see [14, Theorem 18.5]).

The final result of this section gives a characterization of singular linear functionals on $1^{\phi}$.

THEOREM 4.7. Let $f$ be a linear functional on $1^{\phi}$.

(a) The following statements are equivalent:

(1) $f$ is singular.

(2) There exists a unique $v \in B_{\downarrow}(\mathrm{N})$ such that

$$
f(x)=\int x d v \text { for all } x \varepsilon 1^{\dagger} \text {. }
$$

(b) If $f$ is singular, then the following equalities hold:

$$
\bar{\rho}_{\phi}(f)=\|f\|_{\rho_{\phi}}^{*}=\|f\|_{\phi}^{*}-\|f\|_{\phi}^{*}=\|f\|_{\bar{p}_{\phi}}-\left|\|f\|_{\bar{\rho}_{\phi}}-\right| v \mid(\mathrm{N}) \text {. }
$$

PROOF. (a) See the proof of Theorem 4.6. 
(b) According to Theorem 4.6, we get $v_{\mid f}(N)=\mid v_{\lambda}(N)$. Thus, in view of Lemma 4.1, we get

$$
\bar{\rho}_{\phi}(f)=\bar{\rho}_{\phi}(|f|)-\||f|\|_{p_{\phi}}^{*}-\||f|\|_{\phi}^{*}-\|\||f| \|_{\phi}^{*}=\mid v_{\lambda}(\mathrm{N}) \text {. }
$$

Moreover, since $\bar{\rho}_{\phi}(\lambda f)=\bar{\rho}_{\phi}(\lambda|f|)=\lambda \bar{\rho}_{\phi}(f)$ for $\lambda>0$ (see Lemma 4.1), we obtain that $\|f\|_{\bar{p}_{\phi}}=\bar{\rho}_{\phi}(f)$ and $\|f f\|_{p_{\phi}}=\bar{\rho}_{\phi}(f)$. Since the norms which occur in our theorem are Riesz norms the proof is complete.

Since $\left(\left(1^{\dagger}\right)_{s}^{-},\|\|_{0}^{\circ}\right)$ is an abstract L-space (see Corollary 4.2), by Theorems 4.6 and 4.7, we obtain that $B_{q}(\mathrm{~N})$ is also an abstract L-space.

5. The General Form of Continuous Linear Functionals on $1^{\natural}$. We are now in position to give a desired characterization of the dual space $\left(1^{\dagger}\right)^{*}$.

THEOREM 5.1. Let $f$ be a linear functional on $1^{*}$.

(a) The following statements are equivalent:

(1) $f$ is $\tau_{\downarrow}$-continuous.

(2) $f$ is order bounded.

(3) There exist unique $y \in 1^{\circ}$ and $\nu \in B_{\diamond}(N)$ such that

$$
f(x)=\sum_{i=1}^{\infty} x(i) y(i)+\int x d v \text { for all } x \in 1^{\dagger} .
$$

(b) If $f$ is $\tau_{\uparrow}$-continuous, then the following equalities hold:

$$
\begin{gathered}
\bar{\rho}_{\phi}(f)=\rho_{\phi}(y)+|v|(\mathrm{N}), \\
\|f\|_{\phi}^{*}=\|f\|_{\bar{\rho}_{\phi}}=\|y\|_{\phi_{+}}+|v|(\mathrm{N}) .
\end{gathered}
$$

(c) The space $h^{\star}$ is an M-ideal of $\left(1^{\star}, p_{\phi} v\|\|\|\|_{\phi}\right)$.

PROOF. (a) It follows from Theorem 2.4, Theorem 3.2 and Theorem 4.7.

(b) By Theorem 2.4, we have $f=f_{n}+f_{s}$, and it is known that $|f|_{s}-\left|f_{n}\right|,|f|_{s}-\left|f_{s}\right|$, and $\left|f_{n}\right| \wedge\left|f_{s}\right|=0$. Since the conjugate modular $\bar{\rho}_{\phi}$ is orthogonal additive on $\left(1^{*}\right)^{*}$, by Theorem 3.2 and Theorem 4.7, we get $\bar{\rho}_{\phi}(f)=\bar{\rho}_{\phi}\left(f_{n}\right)+\bar{\rho}_{\phi}\left(f_{s}\right)=\rho_{\natural} \cdot(y)+|v|(N)$.

We shall now show that $\|f\|_{+}^{*}-\|y\|_{\varphi^{+}}+|v|(N)$. Indeed, let $\varepsilon>0$ be given. Then there exists $0 \leq x \in 1^{\dagger}$ with $p_{\phi}(x)<1, \rho_{\bar{\phi}}(x)<1$, such that

$$
\left\|f_{n}\right\|_{\leftarrow}^{*}=\left\|\left.\left|f \|_{*}^{*} \leq\right| f\right|_{\infty}(x)+\varepsilon .\right.
$$

Moreover, in view of Lemma 4.1 there exists $0 \leq y \in \omega$ with $\rho_{\uparrow}(y) \leq 1-\rho_{\bar{\phi}}(x)$ such that

$$
\left\|f_{s}\right\|_{\bullet}^{*}-\left\|\left.\left|f \|_{s}^{*} \leq\right| f\right|_{s}(y)\right. \text {. }
$$

Let $z=x \vee y$. Then $\rho_{\bar{\phi}}(z) \leq \rho_{\bar{\phi}}(x)+\rho_{\bar{\phi}}(y) \leq 1$. Moreover, since $p_{\phi}(x)<1$, we have $\rho_{\phi}(x)<\infty$. Hence $\rho_{\phi}(z)<\infty$, so $p_{\phi}(z) \leq 1$. Thus

$$
\begin{aligned}
& \left\|f_{n}\right\|_{\phi}^{*}+\left\|f_{s}\right\|_{\phi}^{*} \leq\left.|f \ln (x)+| f\right|_{s}(y)+\varepsilon \\
& s|f \ln (z)+| f \mid(z)+\varepsilon \\
& -|f|(z)+\varepsilon \leq\|f\|_{i}^{\circ}+\varepsilon \text {. }
\end{aligned}
$$


Hence $\left\|f_{n}\right\|_{\phi}^{*}+\left\|f_{s}\right\|_{\phi}^{*}=\|f\|_{\phi}^{*}$, and, according to Theorem 3.2 and Theorem 4.7, we obtain $\|f\|_{\phi}^{*}=\|y\|_{\phi^{*}}+|v|(\mathrm{N})$. Finally, since $\bar{\rho}_{\phi}\left(\lambda f_{n}\right)=\rho_{\phi}(\lambda y)$ and $\bar{\rho}_{\phi}\left(\lambda f_{s}\right)=\lambda|v|(\mathrm{N})$ for $\lambda>0$, we easily obtain that $\|f\|_{\bar{p}_{\phi}}=\|y\|_{\phi^{*}}+|v|(\mathrm{N})$.

(c) It is well known that $\left(h^{\dagger}\right)^{0}=\left(1^{\dagger}\right)_{s}^{\sim}\left(\right.$ see [26, Theorem 88.10]), where $\left(h^{\phi}\right)^{0}$ denotes the annihilator of $h^{\phi}$ in $\left(1^{\phi}\right)^{*}$. Therefore, from (b) it follows that $\left(h^{\phi}\right)^{0}$ is an L-summand of $\left(\left(1^{\phi}\right)^{*},\|\cdot\|_{\phi}^{*}\right)$ (see [3, Definition 1.1]). According to [3, Definition 2.1] it means that $h^{\phi}$ is an M-ideal of $\left(1^{\phi}, p_{\phi} v\|\cdot\| \|_{\bar{\phi}}\right)$.

REMARK. For a convex Orlicz function $\phi$ the equality $\|f\|_{\phi}^{*}=\|f\|_{\bar{p}_{\phi}}$ has been proved by W. A. Luxemburg and A. C. Zaanen [12, Theorem 5].

As an application of Theorem 5.1 we obtain that continuous linear functionals on $h^{\phi}$ have the unique norm preserving extension to $1^{\phi}$.

COROLLARY 5.3. (see [21, Proposition 3]). Let $g$ be a $\tau_{\phi \mid h^{t^{*}}}$-continuous linear functional on $h^{\phi}$. Then there exists a unique $\tau_{\phi}$-continuous linear functional $f$ on $1^{\phi}$ such that $f(x)=g(x)$ for all $x \varepsilon h^{\phi}$, and $\|g\|_{h^{*}}^{*}=\|f\|_{\phi}^{*}$, where

$$
\|g\|_{h^{+}}^{*}=\sup \left\{|g(x)|: x \in h^{\natural},\|x\|_{\bar{\phi}} \leq 1\right\} .
$$

PROOF. Since $\left(h^{\phi}, \tau_{\phi \mid h^{\dagger}}\right)^{*}=\left(h^{\phi}\right)^{-}=\left(h^{\phi}\right)_{n}^{-}($see [1, Theorem 16.9]), according to [20, Proposition 1.9] and Theorem 3.1 there exists a unique $y \in 1^{*}$ such that $g(x)=\sum_{i=1}^{\infty} x(i) y(i)$ for all $x \in h^{\phi}$. Let us put

$$
f(x)=\sum_{i=1}^{\infty} x(i) y(i) \text { for all } x \in 1^{\phi} .
$$

Then $f(x)=g(x)$ for $x \in h^{\phi}$, and, according to Theorem 3.2,fis order continuous and $\|f\|_{\phi}^{*}=\|y\|_{\phi^{* *} \text {. Now }}$ we shall show that $\|g\|_{h^{*}}^{*}=\|f\|_{\phi}^{*}$. Indeed, we have $\|g\|_{h^{*}}^{*} \leq\|f\|_{\phi^{*}}^{*}$. Let $x \in 1^{\star}$ with $p_{\phi}(x) \leq 1,\|x\|_{\bar{\phi}} \leq 1$. Then

$$
\begin{aligned}
\left|\sum_{i=1}^{\infty} x(i) y(i)\right| & \leq \sup _{n} \sum_{i=1}^{n}|x(i) y(i)| \\
& =\sup _{n} \sum_{i=1}^{\infty}\left|x^{(n)}(i)\right| \cdot \operatorname{sign} y(i) \cdot y(i) \leq\|g\|_{h^{*}}^{*} .
\end{aligned}
$$

Hence $\|f\|_{*}^{*} \leqslant\|g\|_{h^{*}}^{*}$, and we are done.

Now assume that $\bar{f}$ is another such extension of $g$, and let $F=\bar{f}-f$. Then $F$ is singular on $1^{\phi}$ and $\bar{f}=f+F$. Hence, by Theorem 2.4, we have $f=\bar{f}_{n}$ and $F=\bar{f}_{s}$. Therefore, in view of Theorem 5.1, we have $\|\bar{f}\|_{\phi}^{*}=\|f\|_{\phi}^{*}+\|F\|_{\phi}^{*}=\|y\|_{\phi^{*}}+\|F\|_{\phi^{*}}^{*}$ Since $\|\bar{f}\|_{\phi}^{*}=\|g\|_{h^{+}}^{*}=\|y\|_{\phi^{*}}$, we obtain that $F=0$, so $\bar{f}=f$. Thus the proof is completed.

\section{REFERENCES}

[1] ALIPRANTIS, C. D. and BURKINSHAW, O. Locally Solid Riesz Spaces, Academic Press, New York (1978).

[2] ANDO, T. Linear Functionals on Orlicz Spaces, Niew Arch. Wisk. 8 (1960), 1-16.

[3] BEHRENDS, E. M-Strukture and the Banach-Stone Theorem, Springer-Verlag, Lecture Notes in Math. 736, Berlin, Heidelberg, New York, 1979.

[4] BIRNBAUM, Z and ORLICZ, W. Über die verallgemeinerung des begriffes der zueinander potenzen, Studia Math. 3 (1931), 1-67. 
[5] DREWNOWSKI, L. and NAWROCKI, M. On the Mackey Topology of Orlicz Sequence Spaces, Arch. Math. 37 (1981), 256-266.

[6] DUNFORD, N. and SCHWARTZ, J. T. Linear Operators, Part I: General Theory, Interscience, New York, 1958.

[7] FERNANDEZ, R. Characterization of the Dual of an Orlicz Space, Comment. Math, (to appear).

[8] KALTON, N. J. Orlicz Sequence Spaces Without Local Convexity, Math. Proc. Camb. Phil. Soc. 81 (1977), 253-277.

[9] KANTOROVICH, L. V. and AKILOV, G. P. Functional Analysis, Moscow, 1984 (Russian).

[10] KÖTHE, G. Topological Vector Spaces I, Springer, Berlin, Heidelberg, New York, 1983.

[11] KRASNOSELSKII, M. and RUTICHII, YA. B. Convex Functions and Orlicz Spaces. P. Nordhoff Ltd., Groningen, 1961.

[12] LUXEMBURG, W. A. Banach Function Spaces, Delft, 1955.

[13] LUXEMBURG, W. A. and ZAANEN, A. C. Conjugate Spaces of Orlicz Spaces, Indagat. Math. 59 (1956), 217-228.

[14] LUXEMBURG, W. A. and ZAANEN, A. C. Riesz Spaces I, North-Holland Publ. Comp., Amsterdam-London, 1971.

[15] MATUSZEWSKA, W. and ORLICZ, W. A Note on Modular Spaces. IX., ibidem, 16 (1968), 801-808.

[16] NAKANO, H. Modular Semi-ordered Spaces, Maruzen Co. Ltd., Tokyo, 1950.

[17] NAKANO, H. On Generalized Modular Spaces, Studia Math., 31 (1968), 439-449.

[18] NOWAK, M. The Köthe Dual of Orlicz Spaces Without Local Convexity, Mathematica Japonica (to appear).

[19] ORLICZ, W. On Integral Representability of Linear Functions Over the Space of $\phi$-Integrable Functions, Bull. Acad. Polon. Sci. Sér. Sci. Math. Astronom. Phys, 8 (1960), 567-569.

[20] PERESSINI, A. and SHERBERT, D. R. Order Properties of Liner Mappings of Sequence Spaces, Math. Annalen, 165 (1966), 318-332.

[21] RAO, M. M. Linear Functionals on Orlicz Spaces: General Theory, Pacific J. Math. 25 (1968), 553-585.

[22] ROLEWICZ, S. Metric Linear Spaces, Polish Scientific Publishers, Warszawa, D. Reidel Publ. Comp., 1984.

[23] SCHWARZ, H. U. Banach Lattices and Operators, Tuebner-Texte zur Mathematik 71, Leipzig, 1984.

[24] SHAPIRO, J. H. Extension of Linear Functionals on F-spaces, Duke Math. J. 37 (1970), 639-645.

[25] TURPIN, PH. Convexities dans les Espaces Vectoriels Topologiques Generaux, Dissertationes Math. 131 (1976).

[26] ZAANEN, A. C. Riesz Spaces II, North-Holland Publ. Comp., Amsterdam, New York, 1983. 


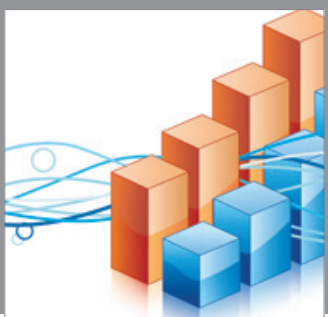

Advances in

Operations Research

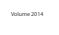

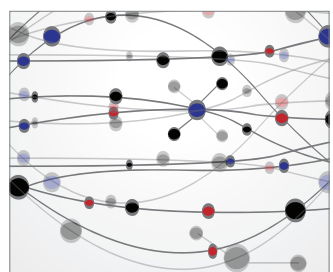

\section{The Scientific} World Journal
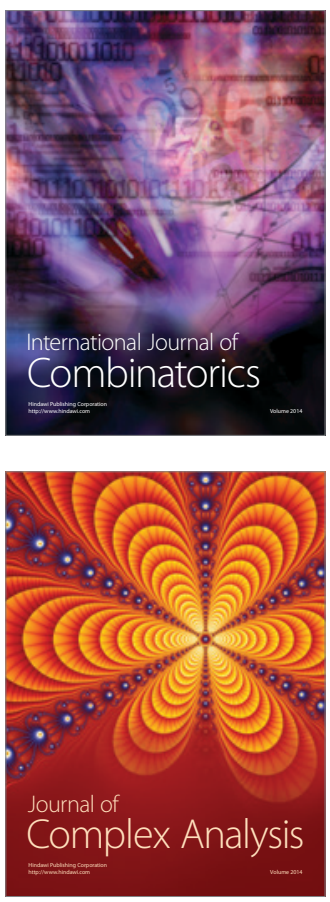

International Journal of

Mathematics and

Mathematical

Sciences
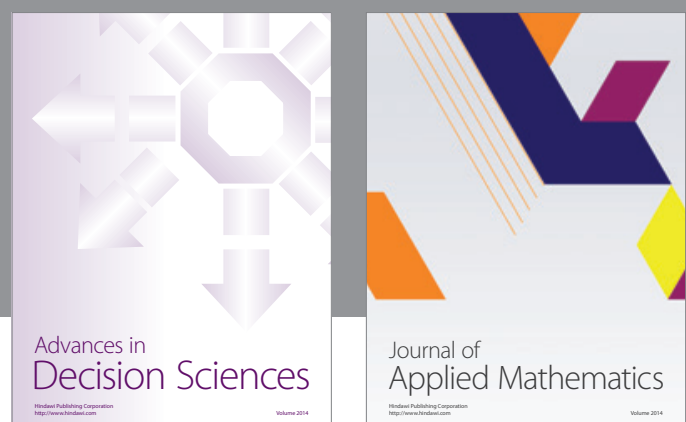

Journal of

Applied Mathematics
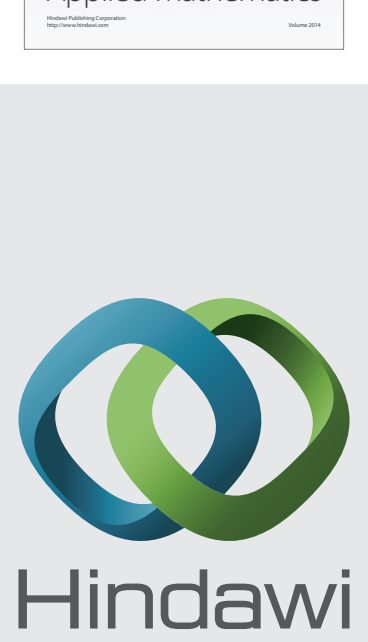

Submit your manuscripts at http://www.hindawi.com
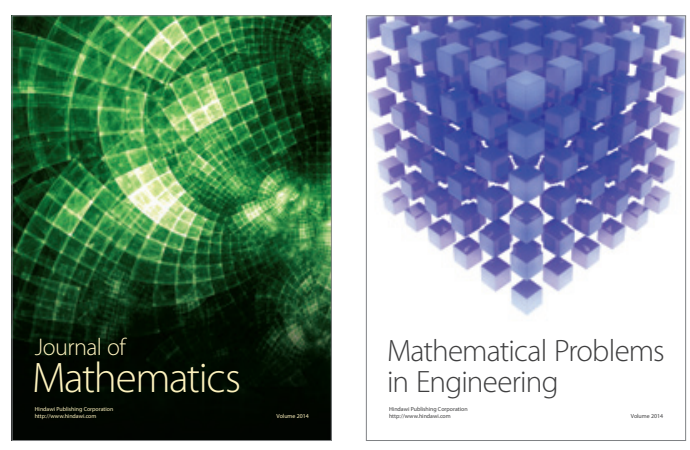

Mathematical Problems in Engineering
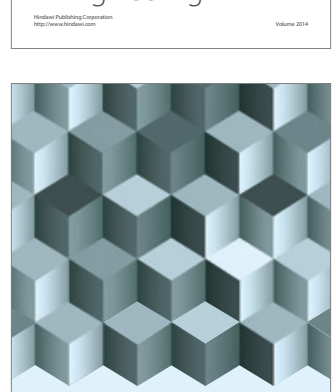

Journal of

Function Spaces
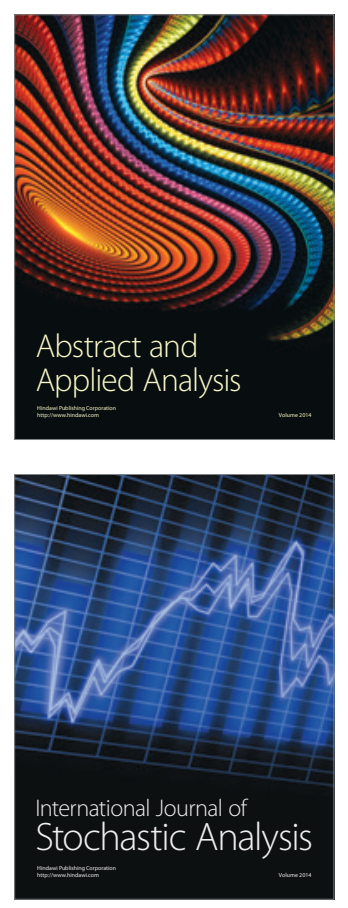

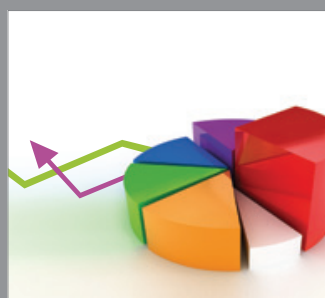

ournal of

Probability and Statistics

Promensencen
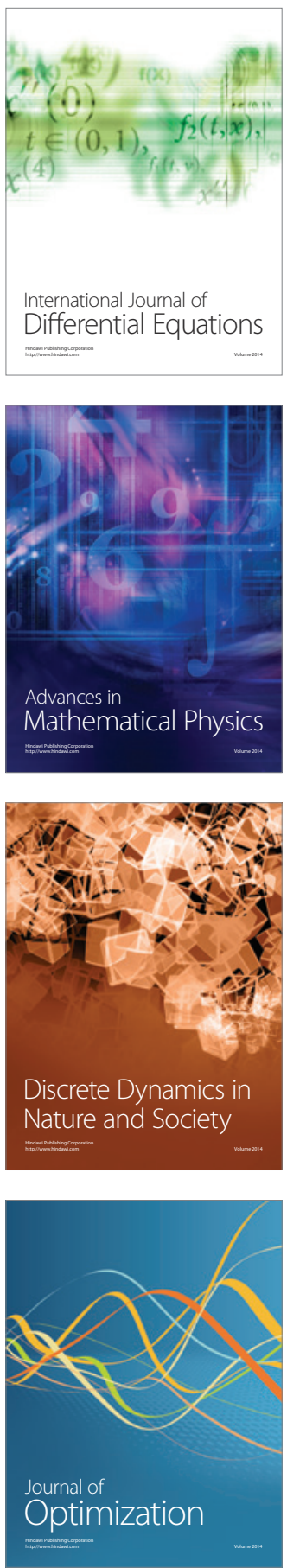